\title{
Guest editorial: special issue on Environmental and Geospatial Data Analytics
}

\author{
Diana Inkpen ${ }^{1} \cdot$ Mathieu Roche ${ }^{2} \cdot$ Maguelonne Teisseire $^{3}$
}

(c) Springer International Publishing AG, part of Springer Nature 2018

Environmental and more generally geospatial information is now provided by crowdsourcing but also by public administrations in the context of the open data policies. Analyses of such data are still challenging, because of their heterogeneity (structural, semantic, spatial, and temporal) and because of the difficulty in choosing the "best" knowledge discovery process to apply, according to the needs of the experts in the field. Challenges about data science deal with creation, storage, search, sharing, modeling, analysis, and visualization of data, information, and knowledge. In the data science context, spatiotemporal aspects are crucial in order to manage and mine data, to index and retrieve information, and finally to discover and visualize knowledge. By taking into account these spatiotemporal aspects, original methods have to be proposed for processing real and complex data from different domains, e.g., environment, agriculture, health, urban, and so forth.

This special issue of the International Journal of Data Science and Analytics Environmental and Geospatial Data Analytics contains a collection of seven papers and provides high-quality research covering part of the challenges mentioned above, from a theoretical or experimental point of view. It includes extended papers from the EnGeoData sessions of DSAA 2015 and DSAA 2016 (IEEE International

Mathieu Roche

mathieu.roche@cirad.fr

Diana Inkpen

Diana.Inkpen@uottawa.ca

Maguelonne Teisseire

maguelonne.teisseire@irstea.fr

1 School of Electrical Engineering and Computer Science, University of Ottawa, Ottawa, ON, Canada

2 French Agricultural Research Centre for International Development (Cirad), TETIS, Univ. Montpellier, APT, Cirad, Cnrs, Irstea, Montpellier, France

3 National Research Institute of Science and Technology for Environment and Agriculture (Irstea), TETIS, Univ. Montpellier, APT, Cirad, Cnrs, Irstea, Montpellier, France
Conference on Data Science and Advanced Analytics) that have been invited to submit.

The EnGeoData sessions bring together researchers interested by pre- and post-processing of environmental data, geographical information retrieval, spatial data mining and spatial data warehousing, knowledge discovery use-cases dedicated to environmental data, spatial text mining, spatial ontology, spatial recommendations and personalization, visual analytics for geospatial data, and dedicated applications.

Geospatial data can be processed by different kind of data mining approaches. The paper of Sujing Wang et al. (A Data Mining Framework for Environmental and Geospatial Data Analysis) deals with a data mining framework, which includes preprocessing of environmental and geospatial data, geospatial data mining techniques, and visual analysis of environmental and geospatial data. The work of Mark P. Wachowiak et al. (Visual analytics of high-frequency lake monitoring data: A case study of multiple stressors on a large inland lake system) focuses on visual analytics techniques. A visual analytics system (i.e., web-based tools) is described that leverages humans' innate capability for pattern recognition and feature detection. The proposed visualizations facilitate community-based participatory research among scientists, government agencies, and community stakeholders.

To extract patterns, considering the complexity of geospatial data, there is a crucial need of specific techniques. In this context, the work of Andrej Dobrkovic et al. (Maritime pattern extraction and route reconstruction from incomplete AIS data) adapts genetic algorithms. The results by comparing with known inland water routes highlight the strengths and weaknesses of the proposed approaches. Following the same way of mining patterns, the paper of Mohomed Shazan Mohomed Jabbar et al. (discovering co-location patterns with aggregated spatial transactions and dependency rules) proposes an original approach to transform spatial data to transaction data by using statistically significant dependency rule searching methods to find co-location rules. The applica- 
tions in environmental health highlight potential associations between air pollution and adverse birth outcomes in Canada.

Finally, data from multi-sources have to be taken into account for analysis of environmental and geospatial data. For instance, a key challenge today consists of handling the domain-specific image streams most efficiently and effectively. In this context, the paper of Keke Chen et al. addresses this issue (SPIN: Cleaning, Monitoring, and Querying Image Streams Generated by Ground-Based Telescopes for Space Situational Awareness). Moreover, other types of data have to be considered like GPS data for trajectory mining. In this context, the paper of Mohamed Quafafou et al. (Detecting Behavior Types of Moving Object Trajectories) computes a formal concept lattice encoding optimal correspondences between hidden patterns and trajectories. Finally, integrating heterogeneous geospatial data is addressed by the hybrid pro- cess of Joo Monteiro et al. (a hybrid approach for the spatial disaggregation of socioeconomic indicators) that takes into account population density, land coverage, nighttime satellite imagery, and OpenStreetMap road density, as ancillary data in order to disaggregate different types of socioeconomic indicators.

All the submitted papers went through the normal journalstyle review process and are finally presented to the readers in the present form. We appreciate the willingness of the authors to help in organizing this special issue. We would like to thank all who submitted papers and all the reviewers for their time, effort, and for completing their assignments on time. 